\title{
Evaluation of Noise Exclusion of Medical Images using Hybridization of Partical Swarm Optimization and Bivariate Shrinkage Methods
}

\author{
Shruti Bhargava*, Ajay Somkuwar** \\ * Department of Electronics and Communication Engineering, Dr. K.N. Modi University, India \\ ** Department of Electronics and Communication Engineering, MANIT Bhopal, India
}

\begin{tabular}{l} 
Article Info \\
Article history: \\
Received Dec 17, 2014 \\
Revised Apr 21, 2015 \\
Accepted May 3, 2015 \\
\hline Keyword: \\
Discrete Wavelet Transform \\
Mean Square Error \\
Peak Signal to Noise Ratio \\
Wavelet De-noising
\end{tabular}

Wavelet De-noising

\section{Corresponding Author:}

Shruti Bhargava,

Department of Electronics and Communication Engineering,

Dr.K.N.Modi University, INS-1, RIICO Industrial Area Ph-II, Newai,

Distt. Tonk, Rajasthan - 304021, India

Email:bhargava.shruti1987@gmail.com
Copyright (C) 2015 Institute of Advanced Engineering and Science. All rights reserved.

\section{INTRODUCTION}

Medical information, composed of images, physiological signals and other clinical data, has become an essential part of a patient's care, during screening, in the diagnostic stage and in the treatment phase. Over the past few decades, there has been a rapid development in information technology (IT) \& Medical Instrumentation which has lead \& facilitated the growth of digital medical imaging. This growth has mainly focused on Computed Tomography (CT), the different digital radiological processes for vascular, nuclear medical imaging with Single Photon Emission Computed Tomography (SPECT), cardiovascular and contrast imaging, mammography, Magnetic Resonance Imaging (MRI), diagnostic ultrasound imaging, and Positron Emission Tomography (PET). All these processes are producing increasing quantities of images. These images are typically different from other photographic images because they reveal internal framework as opposed to an image of external surfaces.

In view of this, survey of literature has been done in the area of tomography, wavelets, multi wavelets and various denoising techniques.. A number of researchers have published image denoising literature [9-25] there is tremendous research that is going on, for better image quality throughout the globe. The thresholding is undertaken on the pixel by pixel basis [26-28] or by considering theinfluence of neighborhood wavelet coefficients on the wavelet coefficients to be thresholded. Cai and Silverman [29] proposed a thresholding method which takes the immediate neighboring coefficients into account to form the threshold. The idea of neighboring wavelet thresholding was extended by Chen and Bui [30] in to the multi waveletscheme. It was proved that neighbor multi wavelet denoising outperforms the neighbor single wavelet 
denoising [31] for some test images and real time signals. Chen et al. [32] proposed a noise suppression method which considers asquare neighborhood window to customize the wavelet filter threshold for image denoising. These methods removethe noises from the images effectively. Crouse et al [33] developed a framework for statistical signal processing basedon wavelet domain hidden markkov models (HMM). Kingsbury [34] proposed the 2D dual tree complex wavelet which satisfies these requirements effectively. But this method is less efficient for motion estimation since the motion information is related to the coefficientphase, which is nonlinear function of estimation.Aim of the paper is to emphasize the problems and solutions in relation to tomographic images which arise in medical field in view of its increasing importance in the present day requirements.

\section{RESEARCH METHOD}

The proposed methodology is basically contains two functional steps

1. generation of initial element

2. determination of fitness function

\section{A. Generation of Initial Element}

In the first process, $n_{a t}$ initial atom, each part of element $n_{E}$ are generated. The set representation of initial elements are given as

$\{R\}_{i i}=\left\{r 0, r 1, r 2 \ldots \mathrm{r}_{\mathrm{nE}-1}\right\}_{i l} ; 0 \leq l \leq n_{a t}-1$

Where $\{\mathrm{R}\}_{\text {il }}$ is the $I^{\text {th }}$ element generated to obtain windows that are closer to the $i^{\text {th }}$ window of the original noisy image. Each atom of the generated element $r_{i l} k \in\{R\}_{i l} ; 0 \leq k \leq n_{E}-1$, is an arbitrary integer generated within the interval $\left[0, \mathrm{n}_{\mathrm{w}}-1\right]$ provided that all the atoms of each element has to satisfy the condition $\mathrm{r} 0 \neq \mathrm{r} 1 \neq \ldots \neq \mathrm{r}_{\mathrm{nE}}-1$.

\section{B. Determination of Fitness Function}

A fitness function decides whether the generated element are fit to survive or not, that can be given as

$$
f_{i}(l)=\frac{1}{n_{c}} \sum_{k=0}^{n_{c}-1} L 2_{i l k}
$$

Wher, $f_{i}(l)$ is the fitness of the $l^{\text {th }}$ element generated for the $i^{\text {th }}$ window $\& L 2_{i l k}$ is the L2 norm distance determined between the $w_{i} \&$ the window indexed by the $k^{\text {th }}$ atom of the $l^{\text {th }}$ element. The $L 2_{i l k}$ is determined as follows

$$
L 2_{i l k}=\sqrt{\sum_{a=0}^{m-1} \sum_{b=0}^{n-1}\left(\left|W_{i}(a, b)-W_{r_{i l k}}^{\prime}(a, b)\right|\right)^{2}}
$$

Where, $W^{\prime} r_{i l k}$ is the window indexed by $r_{i l k}$ that is converted to multi-wavelet transformation domain as done in (4) and (5).

The procedure for wavelet multi resolution transform in is described below

Step 1: At level $j$ 2-D real image is convolved with scale and wavelet filters along the rows of 2-D image.

Step 2: The results obtained after step 1 are convolve again with scale \& wavelet filters along the columns of the 2-D image.

Step 3: Then filter is sub-sampled by a factor of two.

Step 4: At level $j$ the approximation is considered as the input to the next level $j+1$. This procedure is followed for all the levels.

\section{Bivariate Shrinkage Function Model (BFM):}

Bivariate shrinkage function model (BFM) is a new modest non-Gaussian bivariate probability distribution function to perfect the statistics of wavelet coefficients of natural images. The model arrests the dependence amongst a wavelet coefficient \& its parent. Using Bayesian estimation theory we develop from this model a modest non-linear shrinkage function for wavelet denoising, which take a broad view of soft 
thresholding approach. The new shrinkage function, which hangs on both the coefficient \& its parent, produces improved results for wavelet-based image denoising.

Let us consider that w2 symbolize the parent of w1 (w2 is the wavelet coefficient at the identical spatial position as $\mathrm{w} 1$, but then again at the next coarser scale). Then

$$
\mathrm{y}=\mathrm{w}+\mathrm{n}
$$

Where $\mathrm{w}=(\mathrm{w} 1, \mathrm{w} 2), \mathrm{y}=(\mathrm{y} 1, \mathrm{y} 2) \& \mathrm{n}=(\mathrm{n} 1, \mathrm{n} 2)$. The noise standards $\mathrm{n} 1, \mathrm{n} 2$ are iid zero-mean Gaussian with variancelsigma $\mathrm{n}^{2}$.

The standard MAP estimator for $\mathrm{w}$ given the noisy observation $\mathrm{y}$ is:

$$
\hat{w}(y)=\arg \max _{w} \mid P_{w \mid y}(w \mid y)=\arg \max _{w}\left[P_{n}(y-w) P_{w}(w)\right]
$$

The equation for wavelet coefficient $\mathrm{w}_{1}$ is given as

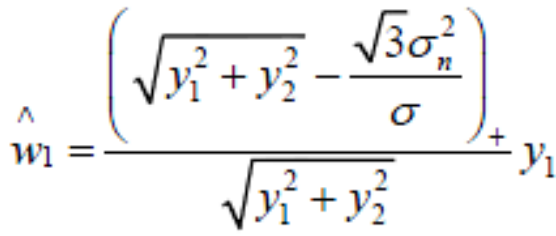

Let consider that

$$
T=\frac{\sqrt{3} \sigma_{n}^{2}}{\sigma}
$$

Then we denote the bivariate shrinkage function model (BFM) $\mathrm{BFM}=\left(Y_{c}, Y_{p}, \sigma_{n}, \sigma\right.$, T), where $Y_{c}$ is the coefficient of each sub-band $\mathrm{Y}_{\mathrm{p}}$ is its parent of the coefficient, $\sigma_{n}$ is the variance of noisy signal, ois the marginal variance $\& T$ is the threshold value.

\section{Methodology for Window Selection in Image Denoising:}

Let, first discuss the already present window selection methodology used in the technique, here, is a brief description.

Let us assume that, I $(\mathrm{x}, \mathrm{y})$ be the unique $\mathrm{CT}$ image and $\mathrm{I}_{\mathrm{AWGN}}(\mathrm{x}, \mathrm{y})$ be the image corrupted by Additive White Gausian Noise, where $0 \leq x \leq M-1,0 \leq y \leq N-1$. The $I_{A W G N}$ is applied to the first stage of the proposed technique, window-based thresholding. The window selection procedure described here is one of the key mechanisms of the first stage of processing of the CT image denoising technique.

In the procedure, a carbon copy of the $\mathrm{I}_{\mathrm{AWGN}}$, labeled as $\mathrm{I}_{\mathrm{AWGN}}$, is generated. $\mathrm{I}_{\mathrm{AWGN}}$ and $\mathrm{I}_{\mathrm{AWGN}}$, a window of pixels are considered \&set to a multi-wavelet transformation. The process of extracting the windows from the image $\mathrm{I}_{\mathrm{AWGN}}$ is given in the Figure 1 . 


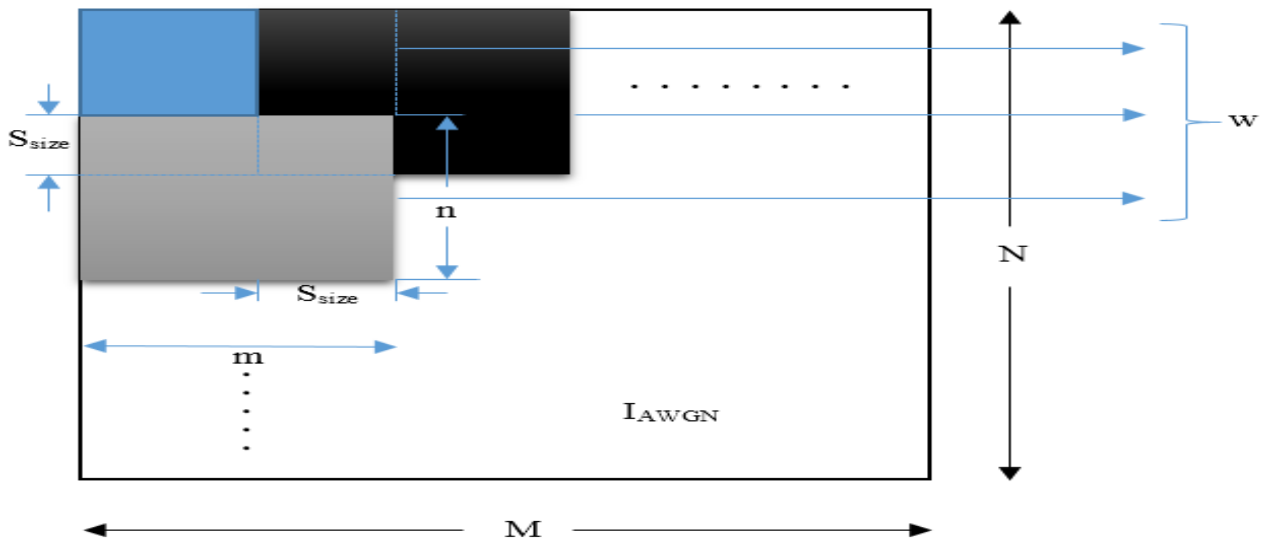

Figure 1. Process of extracting the windows from the given image $\mathrm{I}_{\mathrm{AWGN}}$

In the Figure 1, w indicates the window of pixels pulled out from the image $\mathrm{I}_{\mathrm{AWGN}} \& \mathrm{~S}$ size is the step size of the window. This is carried out all over the image and so $w_{j}$ windows are achieved, where, $0 \leq i \leq n_{w}-1$. By the same way, it is also executed in the image $I_{A W G N}$ and receives $w_{j}, 0 \leq j \leq n_{w}-1$, where, $n_{w}$ signifies number of windows. Then, the obtained window of pixels are transformed to multi wavelet transformation domain as follows

$$
\begin{aligned}
& W_{i}(a, b)=F_{G H M}(a, b) \cdot w_{i}(a, b) \cdot F_{G H M}^{T}(a, b)[30](6) \\
& W_{j}(a, b)=F_{G H M}(a, b) \cdot w_{j}(a, b) \cdot F_{G H M}^{T}(a, b)[50](7)
\end{aligned}
$$

Where, $0 \leq a \leq m-1,0 \leq b \leq n-1$ and $m X n$ indicates the window size. In (6) and (7) $F_{G H M}$ is the concatenated filter coefficient of GHM multi-wavelet transformation, $W_{i}$ and $W_{j}$ are $w_{i}$ and $w_{j}$ in multi-wavelet domain, respectively.

For each $W_{i}, W_{j}$ that are nearer to $W_{i}$ are selected founded on L2 norm distance $\left(L 2_{i j}\right)$, which can be calculated using (9),

$$
L 2_{i j}=\sqrt{\sum_{a=0}^{m-1} \sum_{b=0}^{n-1}\left(\left|W_{i}(a, b)-W_{j}^{\prime}(a, b)\right|\right)^{2}}
$$

Using the $L 2_{i j}$, the $W_{j}$ windows that are nearer to the $W_{i}, W_{L 2 i j}^{c}$ can be demarcated as $W^{\prime}{ }_{L 2 i j}=W_{L 2 i j}-\phi$, where, $W_{L 2 i j}$ is given as

$$
W_{L 2_{i j}}=\left\{\begin{array}{clc}
W_{j}^{\prime} & ; \quad \text { if } \quad L 2_{i j} \leq L 2_{T} \\
\phi \quad & \text { else }
\end{array}\right.
$$

Every $i^{\text {th }}$ window sets in $W^{\prime}{ }_{L 2 i j}$ are organized in ascending order based on their corresponding $L 2_{i j}$. From the sorted window set, $n_{c}$ number of windows are chosen (for every $W_{i}$ ) and the remaining are omitted out, which leads to receive $W$ ' $L 2 i k$, where, $0 \leq k \leq n_{c}-1$.

\section{RESULTS AND ANALYSIS}

With this algorithm subjected to various type of image corrupted by well known type of noises it is being found from the table 2 that all the images has shown a quite improvement in when corrupted by either type of above mentioned noises. Table 1 shows the comparison of our obtained data with the results from WT-TNN approach with db8 and bior6.8 [44] it can be seen that our proposed method has performed well in removing the different type of noises. 


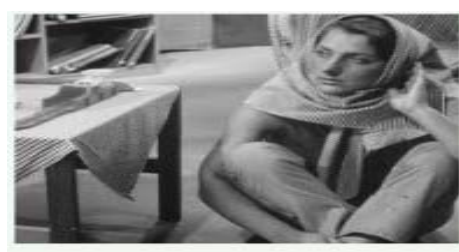

Originat Medicat Image
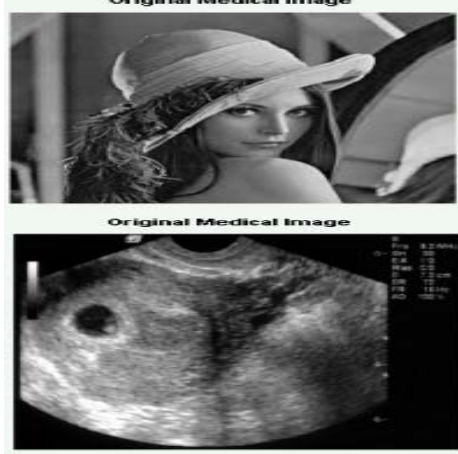

Ortginat medicat tmage

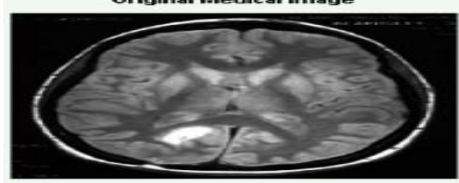

(a)

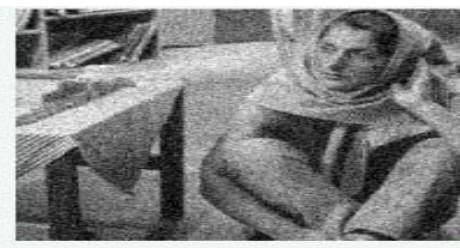

Noised Medicat trmage
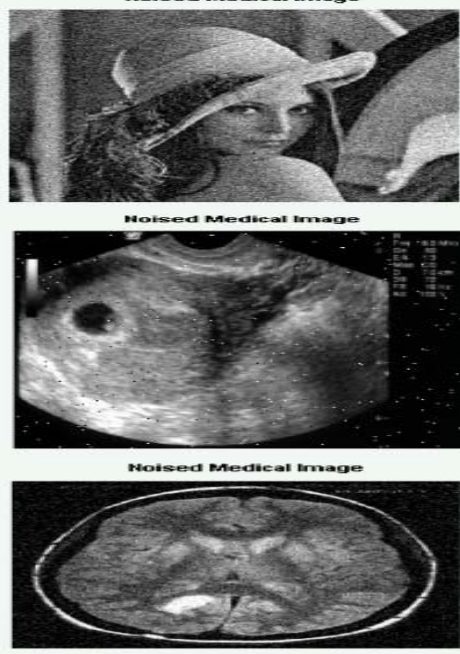

(b)
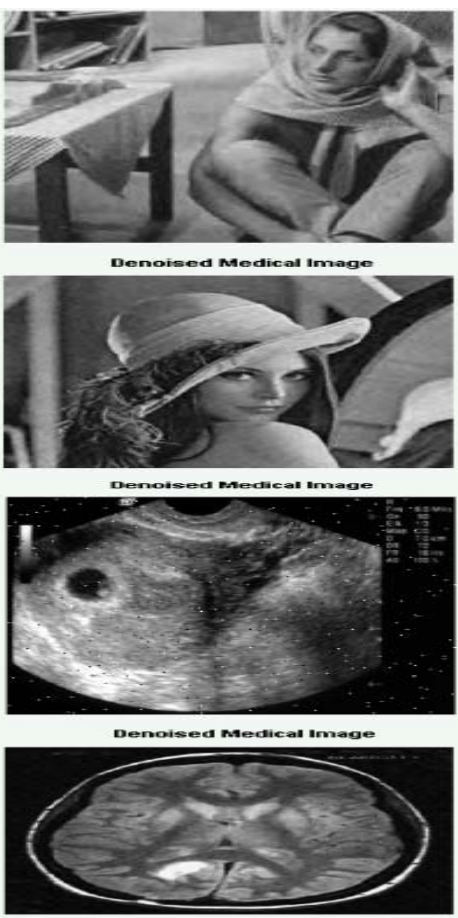

(c)

Figure 2. Image (a) original image, (b) Image with Gaussian noise (c) denoised image

Image denoising using bivariate shrinkage function and which are in turn subjected to Particle Swarm Optimization (PSO) has shown a much significant improvement in salt and pepper noise. Nearly $50 \%$ improvement is seen in PSNR, whereas MSE has decreased to nearly 97\% after adaptive filters. These improvements has helped to achieve better WPSNR between $29 \%$ to $40 \%$ improvement in various image type.Apart from salt and pepper noise, gaussion noise, speckle noise \& poisson noise has shown improvement to some extent but salt and pepper has stood apart from all the noises.

Table 1. Comparative performance of proposed approach with $\mathrm{db} 8$ and bior 6.8 wavelet filter

\begin{tabular}{ccccccc}
\multicolumn{2}{c}{$\begin{array}{c}\text { Set of results from WT-TNN approach with db8 } \\
\text { wavelet filter[44] }\end{array}$} & \multicolumn{3}{c}{$\begin{array}{c}\text { Set of results from WT-TNN } \\
\text { approach with bior6.8[44] }\end{array}$} & \multicolumn{2}{c}{ Proposed with PSO } \\
Image & Noise Std. dev. & PSNR & Noise Std. dev. & PSNR & Noise Std. dev. & PSNR \\
\hline Leena & 10 & 34.29 & 10 & 34.34 & 10 & 35.32 \\
Barbara & 10 & 31.76 & 10 & 31.81 & 10 & 34.3 \\
Ultra Sound & 10 & 33.86 & 10 & 34.64 & 10 & 33.85 \\
\hline
\end{tabular}

Table 2. Performance comparision of proposed methodology and its effect on various noise and image type

\begin{tabular}{|c|c|c|c|c|c|}
\hline Image-Barbara & PSNR & MSE & WPSNR & SSIM & TIME \\
\hline POISSON NOISE & 36.9248 & 13.2008 & 42.1271 & 0.947244 & 0.225509 \\
\hline GAUSSIAN NOISE & 34.3078 & 24.1155 & 37.7427 & 0.88342 & 0.232728 \\
\hline SALT \& PEPPER NOISE & 46.2095 & 17.2363 & 48.8016 & 0.860089 & 0.218133 \\
\hline SPECKLE NOISE & 34.164 & 24.9279 & 39.123 & 0.918154 & 0.248614 \\
\hline Image-Leena & PSNR & MSE & WPSNR & SSIM & TIME \\
\hline POISSON NOISE & 37.8394 & 10.694 & 43.088 & 0.953933 & 0.206691 \\
\hline GAUSSIAN NOISE & 35.3205 & 19.1 & 38.5271 & 0.890729 & 0.212508 \\
\hline SALT \& PEPPER NOISE & 47.0804 & 16.27364 & 48.9249 & 0.834926 & 0.213186 \\
\hline SPECKLE NOISE & 34.7653 & 21.7047 & 39.6693 & 0.923108 & 0.184577 \\
\hline
\end{tabular}




\begin{tabular}{cccccc}
\hline Image-CT Scan & PSNR & MSE & WPSNR & SSIM & TIME \\
\hline POISSON NOISE & 35.0613 & 20.2743 & 40.7958 & 0.954622 & 0.228942 \\
GAUSSIAN NOISE & 34.5149 & 22.9926 & 38.1528 & 0.789865 & 0.208839 \\
SALT \& PEPPER NOISE & 48.8002 & 18.8571 & 43.8698 & 0.828432 & 0.222314 \\
SPECKLE NOISE & 33.7675 & 27.3103 & 38.7148 & 0.949395 & 0.220501 \\
\hline & & & & & SSIM \\
\hline Image-Ultrasound & PSNR & MSE & WPSNR & & TIME \\
\hline POISSON NOISE & 34.8795 & 21.1415 & 40.5439 & 0.961004 & 0.231137 \\
GAUSSIAN NOISE & 33.8518 & 26.7853 & 37.4314 & 0.767568 & 0.211641 \\
SALT \& PEPPER NOISE & 48.5434 & 20.9093 & 42.8133 & 0.840776 & 0.196596 \\
SPECKLE NOISE & 33.3418 & 30.1235 & 37.8567 & 0.950667 & 0.225587 \\
\hline
\end{tabular}

\section{CONCLUSION}

In this paper, new technique has been presented. The proposed Bivariate and PSO based technique approach not only computationally efficient but also gives better performance indicated by performance indices PSNR, MSE, WPSNR, SSIM and time. Finally, it is concluded that the proposed approach in terms of PSNR, WPSNR improvement is outperformed.The proposed technique optimize the possibility of low pass coefficient from each subbandbased on amount of shrinkage is related to signal dependent noise variance.In this paper a new technique is proposed to mitigate the noise in images. According to results the novel Bivariate technique optimized by Particle Swarm Optimization is computationally efficient and performs significantly superior in performance indices indicated by PSNR, MSE, WPSNR, SSIM and time. Finally, we can conclude that in terms of WPSNR and PSNR the proposed approach is outperformed.

\section{REFERENCES}

[1] Arivazhagan S., Deivalakshmi S., and kannan. K.B.N. Gajbhiye, C. Muralidhar, Sijo N. Lukose, M.P. Subramanian, "Performance analysis of wavelet filters for image denoising", Advances in Computational sciences and Technology, vol. 1, no. 1, pp. 1-10, ISSN 0973-6107, 2007.

[2] A.C. Kak and M. Slaney, "Principles of computerized tomographic imaging," IEEE, Inc., New York: IEEE Press. 2006.

[3] Alexander Wong, Akshaya Mishra, Paul Fieguth and David Clausi, 2008, "An adaptive Monte Carlo approach to nonlinear image denoising", Proceedings of the 19th IEEE International Conference on Pattern Recognition, Tampa, pp. 1-4. 1988

[4] Anja Borsdorf, Rainer Raupach, Thomas Flohr and Joachim Hornegger, "Wavelet based noise reduction in CT images using correlation analysis," IEEE Transactions on Medical Imaging, vol. 27, no. 12, pp. 1685-1703. 2008.

[5] Aleksandra Pǐzurica, "A Versatile Wavelet Domain Noise Filtration Technique for Medical Imaging", IEEE Transactions on Medical Imaging, VOL. 22, NO. 3. 2003

[6] Arivazhagan S., Deivalakshmi S., and kannan. K.B.N. Gajbhiye, C. Muralidhar, Sijo N. Lukose, M.P. Subramanian, "Performance analysis of wavelet filters for image denoising", Advances in Computational sciences and Technology, vol. 1, no. 1, pp. 1-10, ISSN 0973-6107, 2007.

[7] Bart Goossens, Aleksandra Pizurica and Wilfried Philips, "Removal of correlated noise by modeling the signal of interest in the wavelet domain", IEEE Transaction on Image Processing, vol. 18, no. 6, pp. 1153-1165. 2009.

[8] Bhatia M. Karl W. and Willsky, A.S.J.P.Morgan \& Co., "Tomographic reconstruction and estimation based on multi-scale natural-pixel bases", IEEE Transactions on Image Processing Vol. 6, Issue 3, pp. 463-478. 1997.

[9] Bing-gang Ye and Xiao-ming Wu, "Wavelet denoising arithmetic research based on small hepatocellular carcinoma CT image", in proceedings of 3rd International Conference on Bio-informatics and Biomedical Engineering, ISBN No: 978-1-4244-2901-1, pp. 1-3. 2009.

[10] D. Gnanadurai and V. Sadasivam, "An efficient adaptive thresholding technique for wavelet based image denoising”, International Journal of Signal Processing, volume 2, number 2, pp. 114-119. 2005.

[11] Detlev Marpe Hans L. Cycon, Gunther Zander and Kai-Uwe Barthel, "Context-based denoising of images using iterative wavelet thresholding", Proc. SPIE Proceedings vol.4671, Visual Communications and Image Processing, C.C.Jay Kuo. Editors, pp. 907-914. 2002.

[12] Dimitrios Charalampidis, "Steerable weighted median filters", IEEE Transactions on Image Processing, vol. 19, no. 4, pp. 882-894. 2010.

[13] D. Donoho, M. Raimondo, "A fast wavelet algorithm for image deblurring", IEEE Transactions on image processing 16 (2). 2006

[14] D.R.K. Brownrigg, The weighted median filter, Communications of the ACM 27 (8) 807-818. 1984

[15] Erdem Bala, Aysin Ertuzun, "A multivariate thresholding technique for image denoising using multiwavelets," EURASIP Journal on Applied Signal Processing, Hindawi Publishing Corporation, vol. no. 2005, issue 8, pp. 12051211. 2005. 
[16] F. Natterer, The mathematics of computerized tomography: Classics-in applied mathematics, SIAM 32, ISBN 0898714931. 2001.

[17] G. Chen, T. Bui, Multiwavelet denoising using neighbouring coefficients, IEEE signal processing letters $10,211-$ 214. 2003.

[18] G. Chen, T. Bui, A.Krzyzak, Image denoising using neighbouring wavelet coefficients, Integrated Computer-Aided Engineering 99-107. 2005.

[19] Hossein Rabbani, "Image denoising in steerable pyramid domain based on a local Laplace prior", Pattern Recognition, vol. 42, no. 9, pp. 2181-2193. 2009.

[20] Hossein Rabbani, "Wavelet-domain medical image denoising using bivariate Laplacian mixure model", IEEE Transactions on Biomedical Engineering, vol. 56, no. 12, pp. 2826-2837. 2009.

[21] Joao M. Sanches, Jacinto C. Nascimento, and Jorge S. Marques, "Medical image noise reduction using the Sylvester-Lyapunov equation”, IEEE Transactions on Image Processing, vol. 17, no. 9, Sept. pp. 1522-1539, 2008.

[22] Landi G. and Loli Piccolomini. E., "An algorithm for image denoising with automatic noise estimation", Journal of Mathematical Imaging and Vision, vol. 34, no. 1, pp. 98-106. 2009.

[23] M. Crouse, R. Nowak, R. Baraniuk, Wavelet based signal processing using hidden markov models, IEEE Transactions on signal processing, 46, 886-902. 1998.

[24] N.P. Anil and S. Natarajan, "A new technique for image denoising using fourth order PDE and Wiener filter", International Journal of Applied Engineering Research, vol. 5, no. 3, pp. 509-516. 2010.

[25] N.T. Binh, A. Khare, Adaptive complex wavelet technique for medical image denoising, ICDBME in Vietnam, IFMBE Proceedings, www.springerlink.com, 27, 196-199. 2010,

[26] Priyam Chatterjee, and Peyman Milanfar, "Is denoising dead?," IEEE Transactions on Image Processing, vol. 19, no. 4, pp. 895-911. 2010.

[27] Prof. S.A. Ali, Dr. S. Vathsal and Dr. K. Lal Kishore, "GA-based Window Selection Methodology toEnhance Window-based Multi-wavelet transformation and thresholding aided CT imagedenoising technique", IJCSIS, Vol. 7, No. 2. 2010.

[28] R. Vijaykumar, P.T. Vanathi, P. Kanagasabapathy and D. Ebenezer, "Robust statistics based algorithm to remove salt and pepper noise in images," International Journal of Signal Processing, vol. no. 5, issue no. 3, pp. 164-173. 2009.

[29] R. Neelamani, H. Choi, R. Baraniuk, Forward: Fourier-wavelet regularized deconvolution for ill-conditioned systems, IEEE Transactions onsignal processing, 52, 418-433. 2004.

[30] R. Silva, R. Minetto, W. Schwartz, H. Pedrini, Adaptive edge-preserving image denoising using wavelet transforms, Pattern Analysis and Applications 1-14doi:10.1007/s10044-012-0266-x.URL http://dx.doi.org/10.1007/s10044-0120266-x, 2012.

[31] S. Arivazhagan, S. Deivalakshmi, K. Kannan, B.N. Gajbhiye, C. Muralidhar, Sijo N. Lukose, M.P. Subramanian, "Performance analysis of image denoising system for different levels of wavelet decomposition", International Journal of Imaging Science and Engineering, GA, USA, vol. 1, no. 3, July, pp. 104-107. 2007.

[32] T. Downie, B.W. Silverman, The discrete multiple wavelet transform and thresholding methods, IEEE transactions on signal processing, 46, 2558-2561. 1998.

[33] T. Cai, B.W. Silverman, Incorporating information on neighbouring coefficients into wavelet estimation, Sankhya: The indian journal of statistics, 63(b), (2), 127-148. 2001.

[34] T.D. Bui, G.Y. Chen, Translation invariant denoising using multivavelets, IEEE transactions on signal processing 46, 3414-3420, 1998.

[35] V. Strela, P. Heller, G. Strang, P. Topiwala, C. Heil, The application of multiwavelet filter banks to image processing, IEEE transactions onimage processing 8, 548-563. 1999.

[36] W. N, W. GT, Wavelet shrinkage and generalized cross validation for image denoising, IEEE Transactions on image processing, 7(1), 82-90. 1998.

[37] Yaniv Gal, "Denoising of Dynamic Contrast-Enhanced MR Images Using Dynamic Nonlocal Means", IEEE Transactions on Medical Imaging, Vol. 29, No. 2. 2010.

[38] Akshaya. K. Mishra, Alexander Wong, David. A. Clausi and Paul. W. Fieguth, "Adaptive nonlinear image denoising and restoration using a co-operative Bayesian estimation approach", Sixth Indian Conference on Computer Vision. 2011.

[39] Francisco Estrada, David Fleet and Allan Jepson, "Stochastic image denoising", British Machine Vision Conference, London 2009.

[40] G. Eason, B. Noble, and I. N. Sneddon. On certain integrals of Lipschitz-Hankel type involving products of Bessel functions [J]. Phil. Trans. Roy. Soc. London. 1995, A247: 529-551.

[41] G.G. Bhutada, R.S. Anand, S.C. Saxena, "PSO based learning of sub-band adaptive thresholding function for image denoising", SIViP (2012) 6: 1-7.

[42] H. Chipman, E. Kolaczyk, R. Mc Culloch, Adaptive bayesian wavelet shrinkage, J Am Stat Assoc, 440(92), 1413$1421,1997$.

[43] J. K. Mandal, S. Mukhopadhyay, A novel technique for removal of random valued impulse noise using all neighbor directional weightedpixels, Proceedings of the International Conference on Commmunications in Computer and Information Science(CCIS), Springer, 203, 102-111. 2011.

[44] J. K. Mandal, S. Mukhopadhyay, Edge preserving restoration of random valued impulse noises (eprrvin), Proceedings of International Conferenceon Recent Trends in Information Systems-RETIS 2011, IEEE 309-314. 2011. 


\section{BIOGRAPHIES OF AUTHORS}

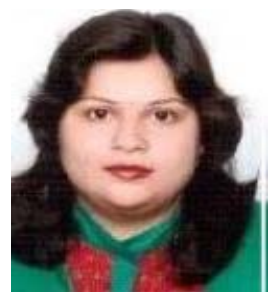

Shruti Bhargava received her Bachelors degree in Electronics and Communication Engineering in the year 2007 and Masters degree in Digital Communication in the year 2010 from R.G.P.V, Bhopal (M.P) India. Currently she is pursing her PhD from the Dr. K.N. Modi University, Newai. Her research interests are Image processing.

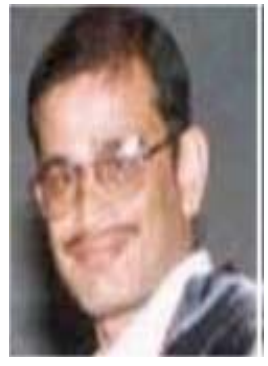

Dr. Ajay Somkuwar received BE with honors from Jabalpur Engineering College and M. Tech. degree in Digital Communication Engineering from MACT Bhopal, subsequently he carried out his research form Indian Institute of Technology, New Delhi and awarded Ph.D. in 2003. Presently he is working as Professor in the Department of Electronics and Communication at Maulana Azad National Institute of Technology, Bhopal. He has published more than 100 papers of national and International repute. He has been Member/ Chairman of many selection committees for recruitment of staff and faculty. His research areas include signal processing Image processing and Biomedical Engineering. He has produced 5 Ph.D. degrees. He is member of IETE, New Delhi and International Association of Engineers (IAENG). Recently he is awarded by "Indira Gandhi Shiromani award-2011" for his contribution to nation. He also awarded by Best Citizen of India-2012. and "Siksha Rattan Award- 2012" 